\title{
Comprehensive analysis of circRNA expression profiles in humans by RAISE
}

\author{
LIN LI $^{1 *}$, YONG-CHANG ZHENG ${ }^{4 *}$, MASOOD UR REHMAN KAYANI ${ }^{1 *}$, WEN XU ${ }^{1 *}$, GUAN-QUN WANG ${ }^{1}$, \\ PEI SUN ${ }^{1}$, NING AO ${ }^{2}$, LI-NA ZHANG ${ }^{3}$, ZHAO-QI GU ${ }^{4}$, LIANG-CAI WU ${ }^{4}$ and HAI-TAO ZHAO ${ }^{4}$ \\ ${ }^{1}$ School of Life Sciences, Center for Synthetic and Systems Biology, Ministry of Education Key Laboratory of Bioinformatics, \\ Collaborative Innovation Center for Diagnosis and Treatment of Infectious Diseases, Tsinghua University, \\ Beijing 100084; ${ }^{2}$ Department of Pathology, Beijing Tiantan Hospital, Capital Medical University, \\ Beijing 100050; ${ }^{3}$ College of Life Science and Bioengineering, Beijing University of Technology, \\ Beijing 100124; ${ }^{4}$ Department of Liver Surgery, Peking Union Medical College Hospital, \\ Chinese Academy of Medical Sciences and Peking Union Medical College, Beijing 100730, P.R. China
}

Received April 5, 2017; Accepted August 25, 2017

DOI: $10.3892 /$ ijo.2017.4162

\begin{abstract}
Circular RNAs (circRNAs) are pervasively expressed circles of non-coding RNAs. Even though many circRNAs have been reported in humans, their expression patterns and functions remain poorly understood. In this study, we employed a pipeline named RAISE to detect circRNAs in RNA-seq data. RAISE can fully characterize circRNA structure and abundance. We evaluated inter-individual variations in circRNA expression in humans by applying this pipeline to numerous non-poly(A)-selected RNA-seq data. We identified 59,128 circRNA candidates in 61 human liver samples, with almost no overlap in the circRNA of the recruited samples. Approximately $89 \%$ of the circRNAs were detected in one or two samples. In comparison, $10 \%$ of the linear mRNAs and non-coding RNAs were detected in each sample. We estimated the variation in other tissues, especially the circRNA highabundance tissues, in advance. Only $0.5 \%$ of the 50,631 brain circRNA candidates were shared among the 30 recruited brain samples, which is similar to the proportion in liver. Moreover, we found inter- and intra-individual diversity in circRNAs expression in the granulocyte RNA-seq data from seven individuals sampled 3 times at one-month intervals. Our findings suggest that careful consideration of inter-individual diversity is required when extensively identifying human circRNAs or proposing their use as potential biomarkers and therapeutic targets in disease.
\end{abstract}

Correspondence to: Dr Yong-Chang Zheng, Department of Liver Surgery, Peking Union Medical College Hospital, Chinese Academy of Medical Sciences and Peking Union Medical College, Beijing 100730, P.R. China

E-mail: liao_medicine@163.com

*Contributed equally

Key words: circRNA, expression, variation

\section{Introduction}

Circular RNAs (circRNAs) are a recently rediscovered class of non-coding RNA $(1,2)$. They are formed by backsplicing events that involve a downstream 3' splice donor site joining an upstream 5' splice acceptor site in the primary transcript. Since the discovery of the first two circular RNAs (DCC in humans and SRY in mice) in the 1990s $(3,4)$, numerous circRNAs have been identified in silico and validated in experiments $(1,2)$. CircRNAs are remarkably stable, conserved, highly abundant and predominantly cytoplasmic (2). They are generated through several distinct mechanisms that rely on complementary sequences within flanking introns $(2,5,6)$, exon skipping $(6,7)$, and exon-containing lariat precursors (8). CircRNA expression is regulated by an RNA editing enzyme or RNA binding proteins, such as ADAR (6) and Quaking (9). Similar to linear RNA, circRNAs are generated from exons or introns at canonical splice sites and require typical spliceosomal machinery (10-12).

Computational biologists have developed several alignment algorithms to identify circRNAs using RNA-seq data. Two main approaches are used to detect circRNAs. One approach uses the annotated genome to build a reference scrambled exon-exon junction database. The scrambled exome includes all possible pairs of intragenic exons in a non-canonical order and the circularization of a single exon. The backsplice junction reads are aligned contiguously along their full length to databases, including KNIFE and other pipelines $(13,14)$. The second strategy improves the alignment algorithms and the pipeline, identifying the backsplice aligned reads to the genome or transcriptome, and examples include mapsplice, find_circ, segemehl, circExplorer, circRNA_finder, CIRI, DCC and acfs (5,15-21). These algorithms differ in accuracy and sensitivity, and there is little overlap in their predictions (22).

Several studies have revealed that circRNAs are substantially enriched in the brain tissues of humans and mice. Of note, the expression levels of circRNAs are dynamic during 
brain development and are independent of the linear transcript that originates from the same gene locus (23-25). In epithelial ovarian carcinoma, circRNAs display altered expression patterns between primary ovarian tumors and metastatic tumors (14). In heart-specific circRNA candidates, there is a lack of differential expression of circRNAs between normal and diseased human heart (26). CircRNAs may also have an impact on aging and multiple disorders. CDR1as (ciRS-7) can serve as a miRNA 'sponge', arresting miR-7 function. In addition, miR-7 is a vital regulatory miRNA in Parkinson's disease (16). Genome-wide association studies linked a newly identified circRNA species, called cANRIL, with atherosclerosis risk (27).

To systematically investigate the intra- and interindividual variation in circRNA expression profiles and the role of circRNAs in humans, we collected a large set of total RNA-seq data from NCBI Sequence Read Archive and ENCODE $(28,29)$. A pipeline named RAISE (circRNA ReAlign Internal Structure and Expression) was developed to analyze circRNA candidates in these samples. Using RAISE, we identified 59,128 circRNA candidates in HCC and adjacent non-tumor tissues. Only a small portion of circRNAs is universally expressed in the recruited HCC samples. The expression of circRNAs in HCC shows inter-individual variations. In advance, we estimated whether circRNA expression varies in other tissues, especially in circRNA high-abundance tissues. Similar to liver cases, only $0.5 \%$ of the 50,631 brain circRNA candidates are shared among the 30 recruited brain samples. Moreover, we found inter- and intra-individual diversity in circRNA expression in granulocyte RNA-seq data from seven individuals sampled 3 times at one-month intervals. Our results suggest that the majority of circRNAs exhibit interand intra-individual variations. When proposing variable circRNAs that are naturally highly expressed as prognostic markers, it is necessary to collect a sufficient number of individuals to confirm that these circRNAs are robustly expressed in humans.

\section{Materials and methods}

RNA-seq datasets. We downloaded publicly available human and mouse RNA-seq data set samples from NCBI SRA (28) and ENCODE (29). Human samples included hepatocellular carcinoma (accession no. GSE65485) (30), granulocyte samples (accession no. GSE70390) (31), brain (accession nos. GSE53697 and GSE71315) (32,33). Mouse samples included liver and brain (accession no. PRJEB5489) (Table I).

RAISE: the workflow of circRNA identification. RAISE is a pipeline designed as a shell script to run after circRNA backsplice sites have been identified. This pipeline can identify circRNA internal structures and alternative exon usage. In this study, we combined four circRNA prediction algorithms, including mapsplice $(2,15,34)$, find_circ (16), acfs $(20,24)$, and circRNA_finder (18). The main steps of RAISE are briefly described in Fig. 1. The first step is to obtain the unmapped reads. RNA-seq reads were aligned to the reference genome and transcriptome by hisat2, filtering contiguous and canonical splice reads. Meanwhile, circRNA_finder was employed to identify circRNAs. The second step is applying mapsplice, acfs and find_circ to detect circRNAs from unmapped reads by combining the three tools and the circRNA_finder results. The third step is to extract the genomic region sequence of the circRNA backsplice site, tandemly duplicating this sequence to create a pseudo circRNA reference. The fourth step is to obtain backsplice junction site coverage and depth. The step 1 unmapped reads are realigned to the pseudo circRNA reference by hisat 2 . The fifth step is conducting paired-end read analysis with one read aligned to the backsplice site. If the mate reads are mapped to the circRNA region, they could be circRNA paired-end reads. If the mate reads are mapped out to a range of circRNA regions, they could be decoys. Then, the proper mapped paired-end reads and decoy reads were counted. The sixth step is to predict the circRNA transcript based on the proper mapped paired-end reads. With one read aligned to the backsplice site, the mate alignment read contains the circRNA inner splice site, which is used to detect circRNA internal structure and exon usage. The alternative step is to detect the support of circRNAs paired-end reads. There are several linear discordant paired-end alignment reads within the genomic region of the circRNA backsplice site that could be the potentially supported reads of circRNA candidates (Fig. 1). This pipeline is available as git repository: https:// github.com/liaoscience/RAISE.

CircRNA annotation and quantification. CircRNA annotation is based on the Gencode (35) human genome (v38) and mouse genome (v10). We intersected the circRNA donator/ acceptor site to annotate gene regions, including coding RNA, non-coding RNA, intron, antisense and intergenic regions, with the BEDTools suite (v2.16.2) (36). The number of reads aligned to the circRNA-specific head-to-tail junctions was used as a measurement of circRNAs expression. Normalization with circRNA Spliced backsplice Reads Per Billion Mapped Reads (SRPBM) in each library was performed to enable the comparison of relative expression among samples (37). Circular to linear ratios were calculated using the backsplice reads of circRNAs by dividing the mean value of reads that span the linear splice junction reads, including the left and right sides of the circRNA splice sites (38).

Mapping and quantification of linear mRNA and lncRNA expression. Sequencing quality was assessed by FASTQC (39). After removing adaptor and low-quality reads using cutadapt (40) (-q 10 -e $0.1-\mathrm{O} 10-\mathrm{m} 50$ ), the clean reads were aligned to the human (hg38) genome reference sequences using hisat2 (41) with the default parameters. The bam files were generated, sorted, and deduplicated using SAMTOOLS (v1.3) (42). Read counts were tabulated with HT-Seq (43) in 'union' mode with the Gencode human v24 GTF file as a reference. Stringtie (v1.3) (44) was also used to estimate the total transcriptional output based on the Gencode human gene annotation (HG38 version 24) (35).

Gene Ontology enrichment analysis. Gene ontology (GO) term enrichment analysis was performed using DAVID (45), by inputting the list of circRNA derived host locus genes.

Statistical analysis. The raw counts were first normalized using trimmed mean of M-values (TMM). Differential 
Table I. Summary of RNA-seq data set.

\begin{tabular}{lrccccl}
\hline Accession no. & Liver & Brain & Granulocytes & Other & Total & Description \\
\hline GSE65485 & 55 & 0 & 0 & 0 & 55 & 50 HCC and 5 adjacent non-tumor \\
GSE77661 & 4 & 2 & 0 & 20 & 26 & In 4 liver samples, 2 are normal, 1 is HCC, \\
& & & & & 1 is HCC adjacent non-tumor tissue, 2 heart \\
GSE73570 & 0 & 0 & 0 & 6 & 6 & Blood 1 repeat \\
encode & 2 & 3 & 0 & 38 & 43 & 2 liver sample, 3 brain samples \\
GSE53697 & 0 & 17 & 0 & 0 & 17 & 8 control, 9 Alzheimer's diseased \\
GSE71315 & 16 & 0 & 0 & 16 & 8 ribozero total RNA, 8 polyA RNA \\
PRJEB5489 & 12 & 12 & 0 & 0 & 21 & Mouse normal \\
GSE70390 & 0 & 0 & 21 & Human granulocytes 7x3
\end{tabular}

circRNA or gene expression was estimated using the edgeR package, and a negative binomial model was used to estimate differential expression between tumor and adjacent non-tumor tissues (FDR <0.02, 2-fold change) (46). Statistical analyses were performed using R 3.3.1 (http://www.r-project.org/).

\section{Results}

RAISE: a cocktail of circRNA analysis pipeline. Numerous computational pipelines use backsplice reads to identify circRNAs; however, there is little overlap in these circRNA detection methods. Each algorithm has bias and 'blind spots', so we combined several different read aligners to identify more circRNAs and increase the robustness of circRNA identification (22). Previous algorithms detect circRNA backsplice sites, but they do not include internal structure information. We combined four available circRNA detection algorithms (mapsplice, acfs, circRNA_finder, and find_circ) to develop an integrated pipeline called RAISE to improve the prediction accuracy and detect the internal exon usage of circRNAs (Fig. 1). RAISE is an easy-to-use shell script pipeline. The four selected tools chosen are based on previous reviews and research $(22,34)$.

We tested RAISE on human liver rRNA depleted samples from ENCODE. In this RNA-seq library, 5,977 circRNA candidates were detected by mapsplice, 6,672 circRNA candidates by acfs, 10,778 circRNA candidates by circRNA_finder, and 8,952 circRNA candidates by find_circ. There were 2,891 circRNA candidates that were detected by all four tools. After application of the RAISE pipeline, 14,145 circRNA candidates were detected, and when we filtered out candidates with less than two backsplice reads, there were 8,270 circRNA candidates for advanced analysis. We compared the abundance of circRNA candidates between these tools and RAISE. The abundance in acfs and mapsplice was close to that of RAISE, whereas the abundance in find_circ and circRNA_finder was less than that in RAISE (Fig. 2A and B). Furthermore, the internal exon usage of 3,052 high-abundance circRNA candidates was predicted. For example, the exon composition of circABCB4 contains exon 13 and exon 14 of the ABCB transcript, with one read aligned to the backsplice sites and the mate alignment read containing cis-junction splice information, which is consis- tent with the linear RNA junction site (Fig. 2C). A total of 1,255 alternative splice sites were also detected in the library; for example, circPLOD2 was derived from exons 2 and 3 of the PLOD2 gene, and there were two cis-splice junctions in intron 2 (Fig. 2D). Exon usage was different with the host locus linear RNAs.

CircRNAs display only minor alterations in expression in HCC and adjacent non-tumor tissue. In order to investigate the circRNA expression profile in HCC, we collected 61 human liver samples. All of the RNA-seq data are non-poly(A)-selected, and are downloaded from NCBI and ENCODE, GSE65485 included 50 HCC samples and 5 adjacent non-tumor tissues; GSE77661 included 4 liver samples; ENCODE included 2 liver samples (Table I). These data included $51 \mathrm{HCC}$ samples, 6 adjacent non-tumor samples and 4 normal liver samples. The sequencing depths of these samples ranged from 29.3 to 122.3 million reads. Approximately $95 \%$ of these reads were aligned to the human reference genome (hg38). Multidimensional scaling (MDS) (47) analysis showed that the HCC samples were distinct from normal and adjacent non-cancerous tissue samples (Fig. 3A).

The RAISE pipeline was applied on the unmapped reads (5\%), and 59,128 distinct circRNA candidates were identified in 61 liver samples. We chose seven HCC samples and five adjacent non-tumor samples (unpaired samples) to compare the expression patterns of circRNAs between tumor and adjacent non-tumor tissues. First, we found that $80 \%$ of the circRNA candidates in these $12 \mathrm{HCC}$ samples were derived from the protein-coding exonic regions while other smaller fractions were antisense, long non-coding RNAs, intergenic regions and intronic regions (Fig. 4A). The genomic features of these circRNAs, e.g., genomic origins, exon numbers, exon length and genomic distance, were compared between HCC and adjacent non-tumor tissue. The exon numbers of most circRNAs were less than five. The length of most exonic circRNAs was $\sim 300-500 \mathrm{nt}$ with a genomic distance of $\sim 1,000-3,000 \mathrm{bp}$. There was no significant difference in the genomic features between $\mathrm{HCC}$ and adjacent non-tumor tissue (Fig. 4B-D).

Next, we inquired whether liver circRNAs are differentially expressed between tumor and adjacent non-tumor tissues. Unlike mRNAs and lncRNAs, hundreds of linear RNA genes are significantly differentially expressed in these 


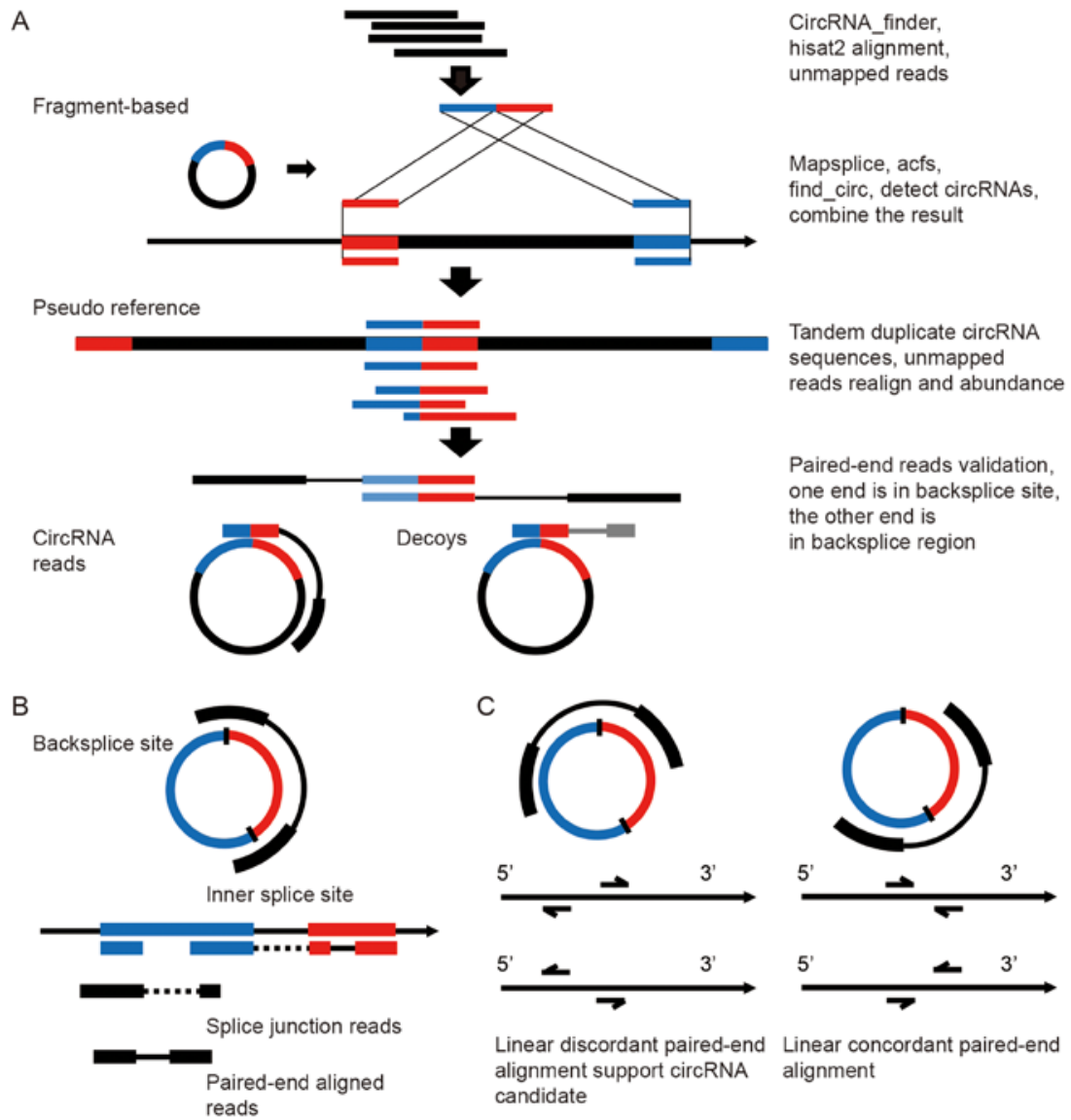

Figure 1. The RAISE workflow for the identification and quantification of circRNA candidates. (A) The pipeline of circRNA detection: 1, Hisat2 alignment and filtering of mapped reads, STAR alignment and circRNA_finder identification. 2, Detection of circRNAs from unmapped reads using mapsplice, acfs and find_circ tools, and merger of their outputs with the circRNA_finder results. 3, Extraction of the genomic region corresponding to the circRNA backsplice site from the previous four tools' output, tandem duplication of the sequences to create a pseudo circRNA reference, realignment of the unmapped reads to the pseudo reference, and estimation of the abundance of circRNA candidates. 4, From the paired-end data, if one read is aligned to a circRNA backsplice site and if the mate read is aligned to the circRNA region, these paired-end reads are classified among circRNA reads, whereas if the mate read is aligned outside the circRNA backsplice site, the paired-reads are classified as decoys. (B) Prediction of circRNA internal exon usage by paired-end and splice junction reads. (C) Discordant linear alignment reads that could be potential circRNA candidate paired-end reads.

A

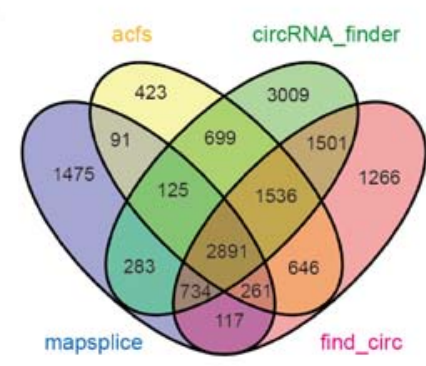

C

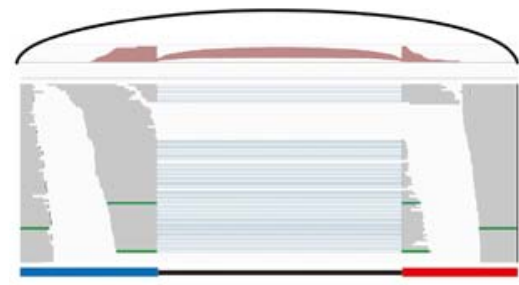

CircABCB4 internal structure (exon 13-14)

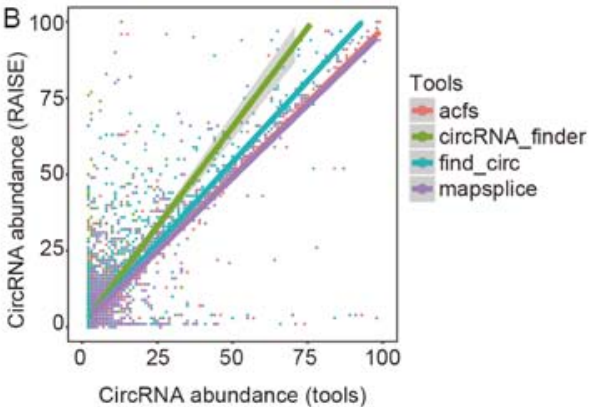

D

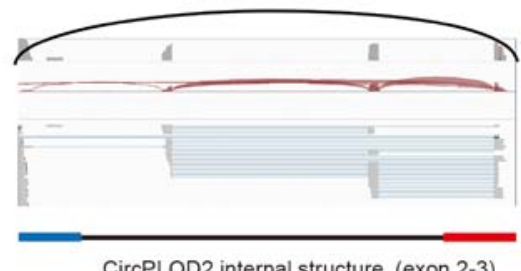

CircPLOD2 internal structure (exon 2-3)

Figure 2. Summary of RAISE prediction results. (A) Venn diagram comparison of circRNAs identified by the four tools. (B) Scatter plot of circRNA abundance between RAISE and the four tools. (C) The internal structure is the same as that of linear RNAs. (D) The internal structure is different from that of linear RNAs. CircRNA internal structure displayed by IGV. Red and blue bold lines indicate exons, a curved red line indicates the $c i s$-splice junction of the circRNA, a curved black line indicates the backsplice junction of the circRNA, gray and green lines indicate the paired-end alignment reads, and gray peaks indicate the read depth in the genomic region. 
A

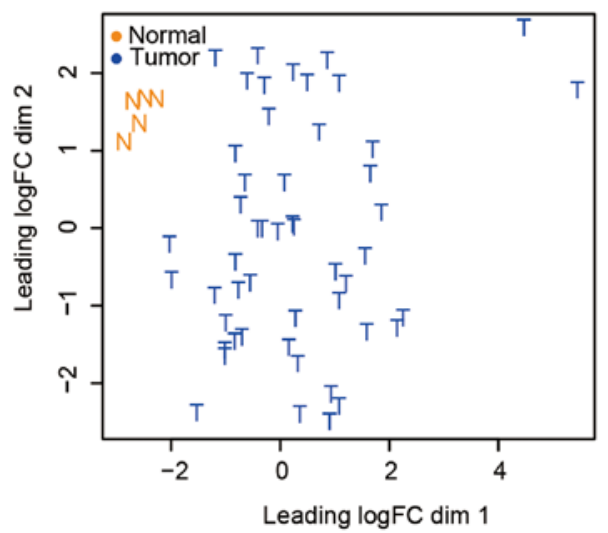

B MDS of $\mathrm{HCC} 55$ samples circRNA transcripts

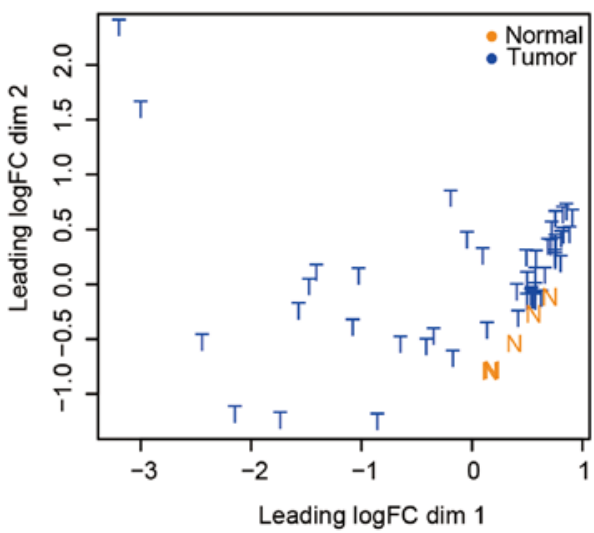

C
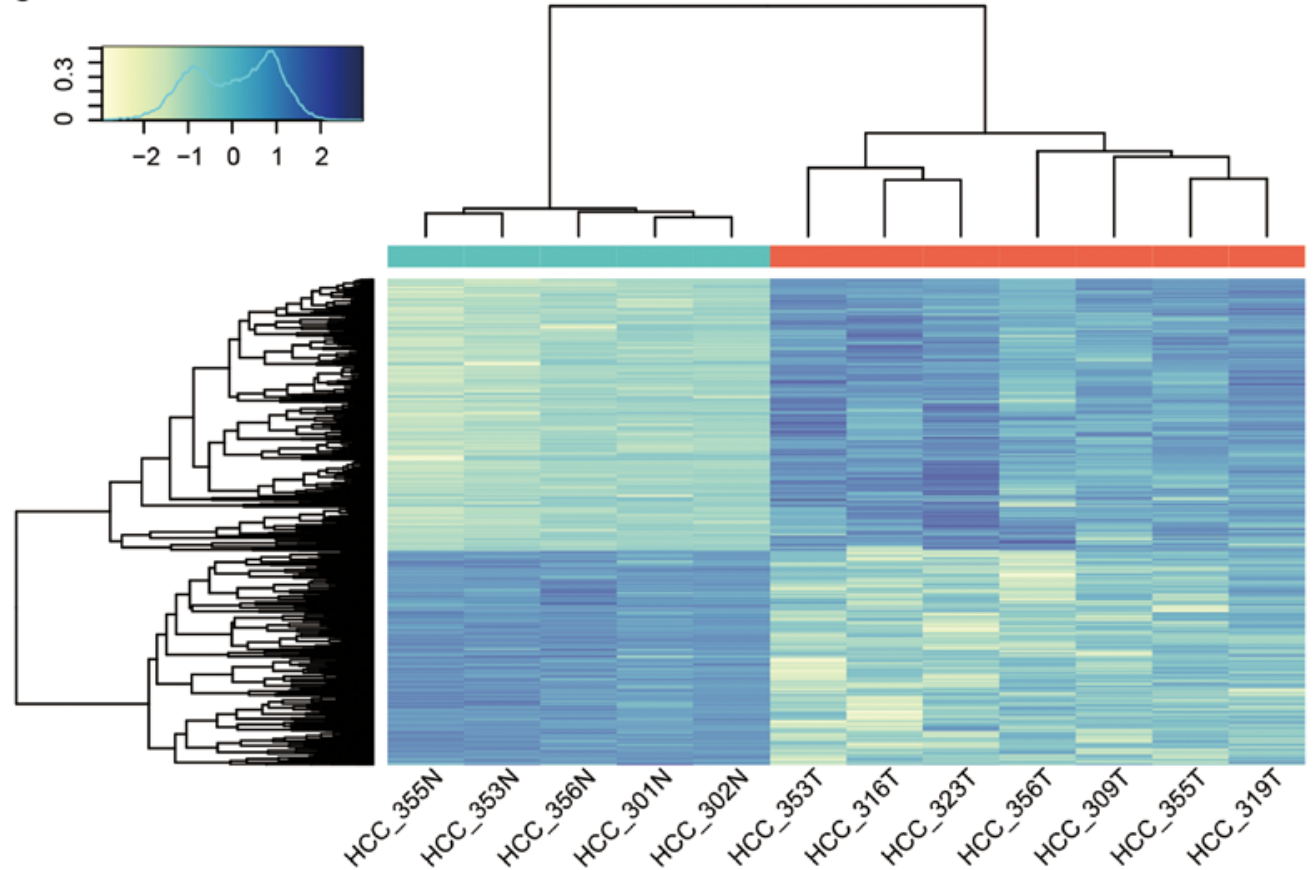

Figure 3. Multidimensional scaling (MDS) analysis of the $55 \mathrm{HCC}$ samples. (A) Linear transcriptome. (B) CircRNAs transcriptome. (C) A heatmap displaying the differentially expressed linear gene abundance in 12 HCC samples.

two groups. We did not detect any differentially expressed circRNA candidates with statistical significance (Fig. 4E). The circRNA circALB (chr4:73405119-73408712) from exon 2-4 of the ALB gene was in high abundance in adjacent non-tumor tissue, but it was not detected in $84 \%$ of a total of $50 \mathrm{HCC}$ samples. Of note, ALB mRNA was highly abundant in the liver samples.

CircRNAs show inter- and intra-individual expression diversity. In addition to assessing circRNA expression patterns in HCC, we turned our attention to the liver-specific expression of circRNAs. We calculated the occurrence of each circRNA in these samples to discover the shared circRNAs and unique circRNAs. For example, circAPOA2 was detected in $28 \mathrm{HCC}$ samples; hence, its occurrence was 28. In 55 HCC samples, the occurrence of circRNAs ranged from 1 to 55 . We analyzed the total detected circRNA candidates and linear gene occurrences. Unlike linear RNAs, $\sim 10 \%$ of the protein-coding RNAs and non-coding RNAs were expressed in all 55 HCC samples, whereas almost no detected circRNAs were shared by the 55 samples (Fig. 5A). Consequently, a single gene locus can transcribe multiple circular isoforms $(48,49)$. We asked whether the diversity among the samples is due to variations in circular RNA isoform selection within the gene locus. We set the circRNAs derived from the same transcript as 'transcript circRNA' and those from the same gene locus as 'gene circRNA'. For example, there are 329 circRNA isoforms in the ALB gene locus; we set these circRNAs as circALB. Furthermore, we compared the diversity in geneand transcript-level circular RNA expression. Seven of the 16,133 transcript circRNAs and 9 of the 9,696 gene circRNAs were shared among the $55 \mathrm{HCC}$ samples (data not shown). These nine genes expressed circRNAs in all the samples independently of the circRNA backsplice sites. The ratio of transcript to gene circRNAs remained low in contrast to the ratio of transcript to linear RNAs. To test whether a similar expression pattern exists in mice, we downloaded 12 mouse liver RNA-seq datasets from NCBI SRA, analyzed them with 
A

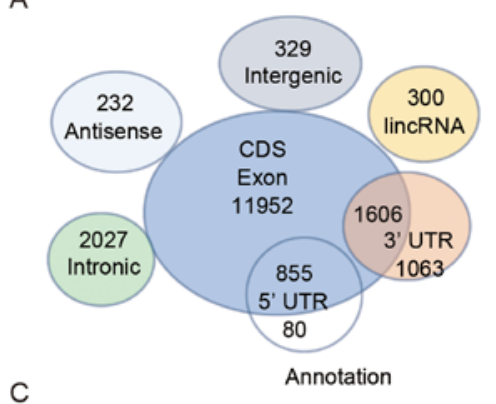

$\mathrm{C}$

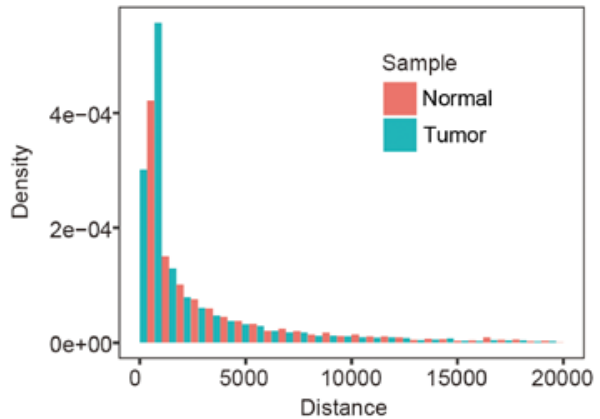

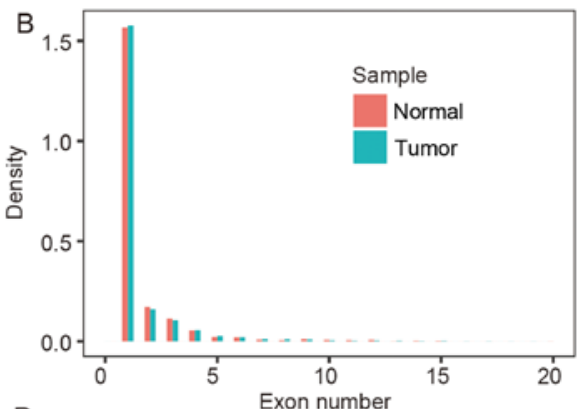

D

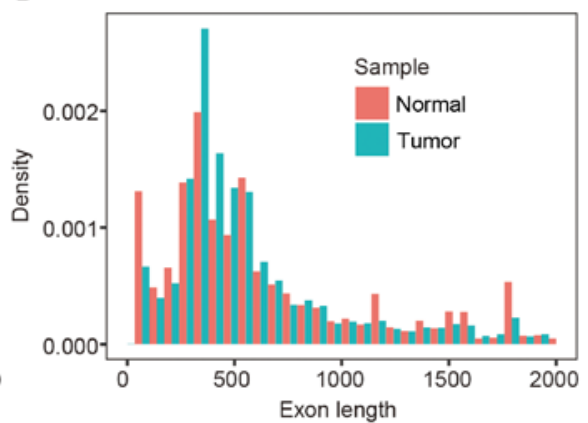

E

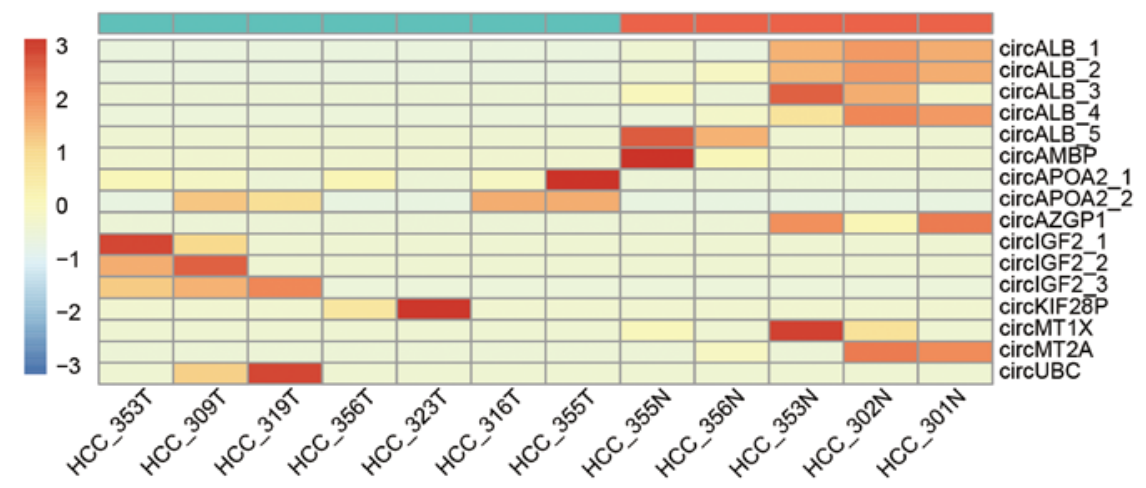

Figure 4. CircRNAs display no difference between HCC and adjacent non-tumor tissue. (A) The genomic origin of circRNAs. (B) The exon number distribution of circRNAs. (C) The genomic distance of backsplice sites. (D) The length distribution of exonic circRNAs. Tumor represents HCC. Normal represents adjacent non-tumor tissue. (E) Heatmap of several circRNA expression profiles from the samples. N represents adjacent non-tumor tissue. T represents HCC.

the same pipeline (Table I), and identified 3,801 circRNA candidates in these samples. Only $0.1 \%$ of circRNAs were shared in the mouse liver samples, i.e., $0.1 \%$ of the transcripts as well as $0.1 \%$ of the gene circRNAs were shared in each sample. This result is consistent with the human liver circRNA expression profile (Fig. 5B).

We then asked whether inter-individual circRNA expression diversity is also common in other human tissues. Since circRNAs are highly abundant in the brain (data not shown) (24), we downloaded several batches of brain ribosomal RNA depleted RNA-seq data from NCBI SRA and analyzed the brain circRNA expression profile with our pipeline (Table I). We identified 50,631 circRNA candidates in 30 human brain samples and found $\sim 0.5 \%$ shared in each brain sample (Fig. 5C). The same pipeline was used on the mouse brain samples, and its results showed similarities to those of the human brain (Fig. 5D). Circular RNAs display greater variation than linear genes in both brain and liver.

CircRNA expression profiles are varied and diverse between individuals, whether or not circRNAs are reproducibly expressed within one individual. We downloaded
21 human granulocyte ribo-zero RNA-seq datasets from NCBI SRA (Table I). The data came from seven healthy individuals, with three samples at least one month apart (31). We used the previous pipeline to identify circRNAs and found that $3 \%$ were shared among these 21 samples. Within the individuals, circRNAs showed less reproducible expression than linear RNAs between different individuals (Fig. 5E and Table II). The unsupervised hierarchical clustering of the circRNA expression profiles of the 21 samples displayed inconsistent results within each donor (Fig. 6).

In short, for the tissues with low circRNA abundance, e.g., liver, the ratio of shared circRNAs was lower. For the tissues with high circRNA abundance, e.g., brain and granulocytes, the ratio increased.

Shared circRNAs are highly abundant and are derived from circRNA hotspot gene loci. We further investigated the characteristics of shared circRNAs and unique circRNAs. We observed that with an increase in the occurrence of circRNAs, their abundance also increased in all the studied samples. In $13 \mathrm{HCC}$ and 17 brain samples, the abundance of the highest 
A 55 human HCC samples
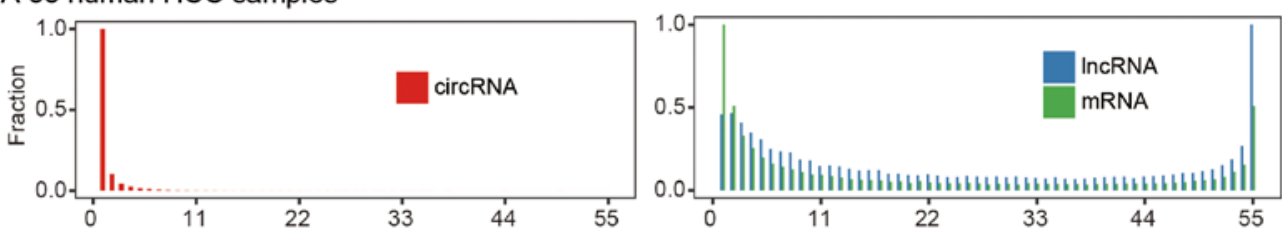

B 12 mouse liver samples
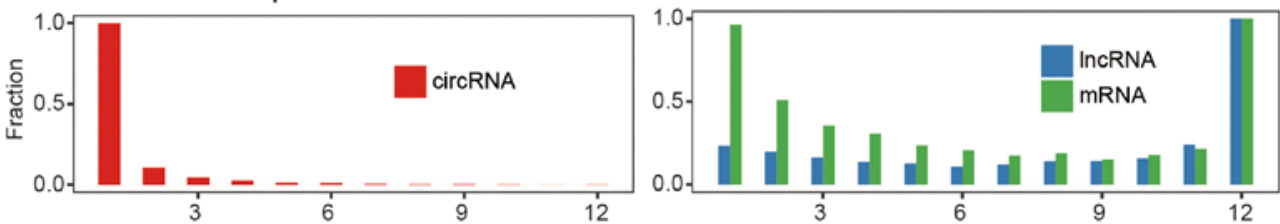

C 30 human brain samples
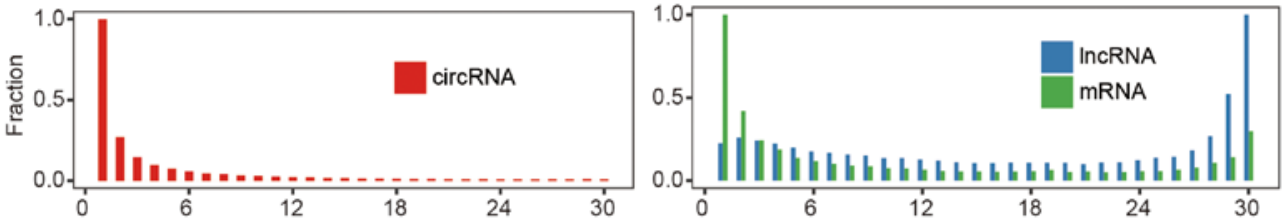

D 12 mouse brain samples
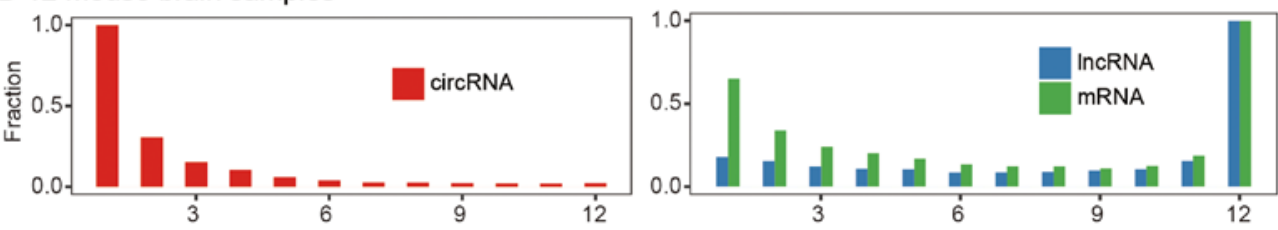

E 21 human granulocyte samples
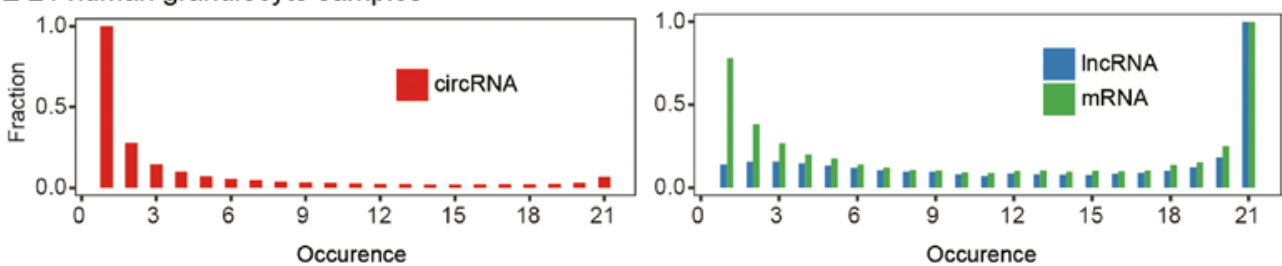

Figure 5. Comparison of circRNA and linear RNA distributions in humans and mice. x-axis corresponds to the occurrence of RNA in the recruited samples; y-axis represents the fraction of RNAs. (A) Fifty-five human HCC samples. (B) Twelve mouse liver samples. (C) Thirty human brain samples. (D) Twelve mouse brain samples. (E) Twenty-one human granulocyte samples.

Table II. CircRNA distribution in different tissues.

\begin{tabular}{lrrrrr}
\hline Specie tissue & Human brain & Mouse brain & Human liver & Mouse liver & Human granulocytes \\
\hline Samples number & 30 & 12 & 61 & 12 & 21 \\
CircRNA candidates & 50,631 & 7,124 & 59,128 & 3,801 & 31,063 \\
Prediomant & 5,017 & 516 & 2,743 & 244 & 3,893 \\
Alter splice & 3,042 & 182 & 5,228 & 55 & 1,528 \\
Shared (>20\%) & 8,910 & 1,983 & 469 & 353 & 8,509 \\
Shared prediomant & 405 & 157 & 8 & 19 & 984 \\
Shared alter splice & 232 & 33 & 3 & 4 & 268 \\
Unique (<20\%) & 41,721 & 5,141 & 58,659 & 3,448 & 2,909 \\
Unique prediomant & 4,612 & 359 & 2,735 & 225 & 1,318 \\
Unique alter splice & 2,869 & 156 & 5,224 & 55 & 12,809 \\
Transcript circRNA & 19,544 & 4,177 & 20,530 & 2,788 & 4,584 \\
Co-exist & 8,086 & 1,695 & 8,516 & 1,291 & 1,849 \\
Shared co-exist & 2,082 & 634 & 333 & 192 & \\
\hline
\end{tabular}

Shared circRNAs is the occurence of circRNAs in $>20 \%$ of the recruited samples. Unique circRNAs is the total circRNA candidates minus the shared circRNAs. Prediomant circRNAs is in the circRNA backsplice sites, when backsplice reads of circRNAs more than the reads that span linear splice junction reads. Alter splice is the circRNAs internal structure which is different from linear RNAs. Transcript circRNA refers to the circRNAs reference transcripts. Co-exist is circRNAs and linear RNA genes co-expression. 


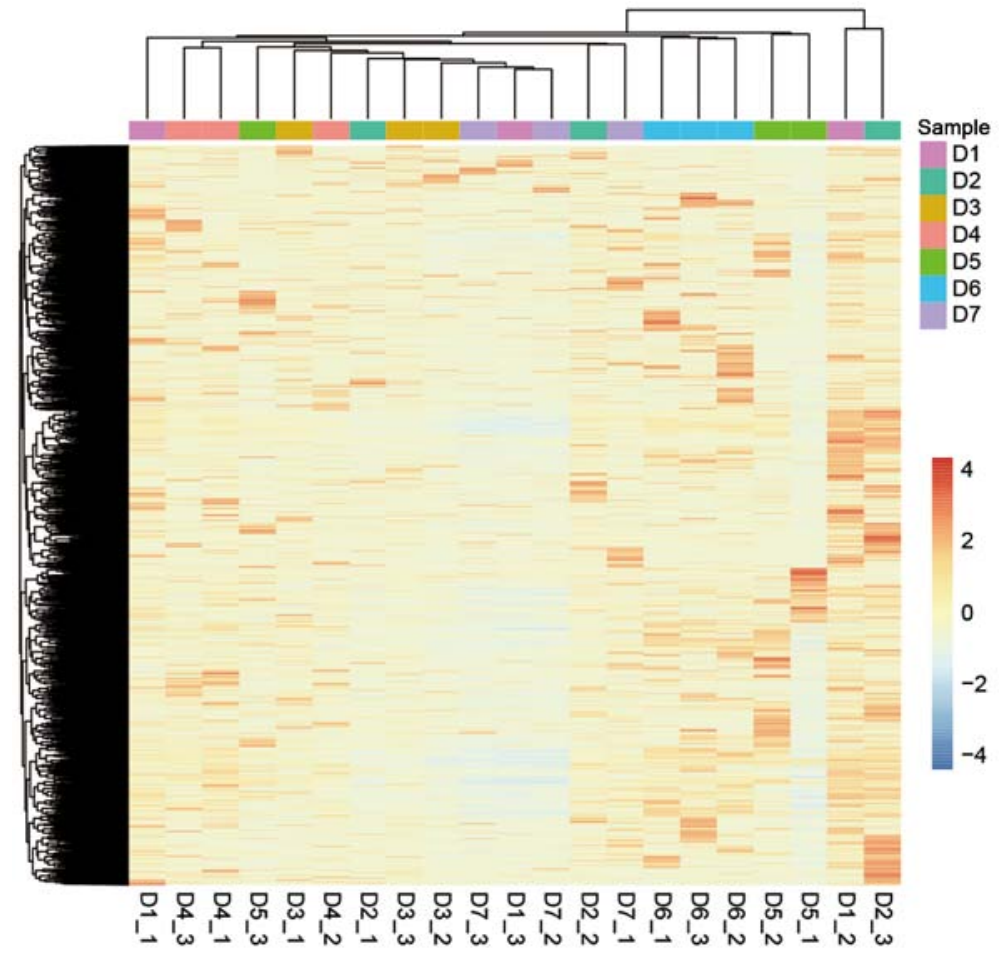

Figure 6. CircRNA expression displays inter- and intra-individual diversity in human granulocyte samples (seven individuals, sampled at three time-points spaced at least 1 month apart). Unsupervised hierarchical clustering of the circRNAs. Heatmap colors represent relative circRNA abundance in each sample.

\section{A 13 human HCC samples}

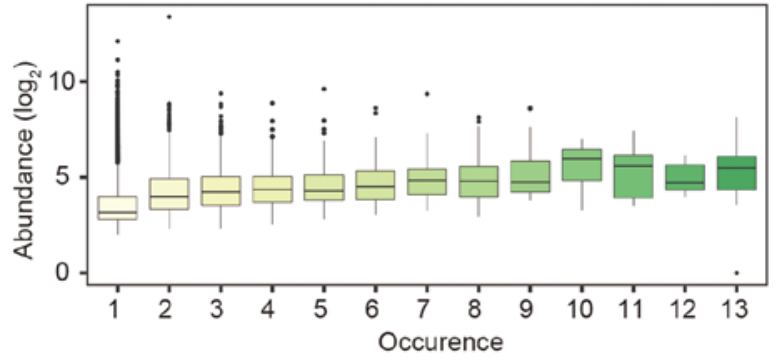

C 17 human brain samples

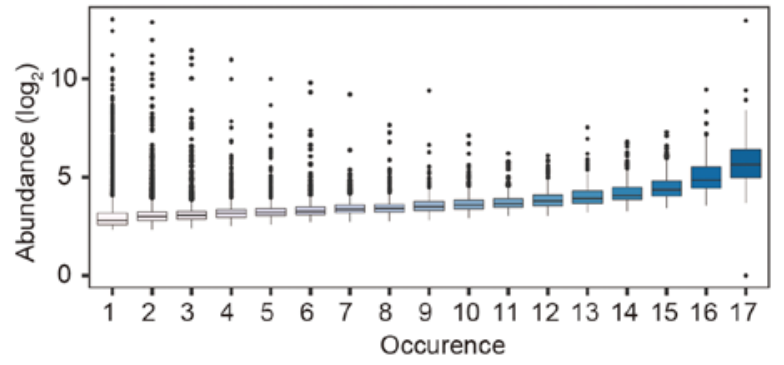

E 21 human granulocyte samples

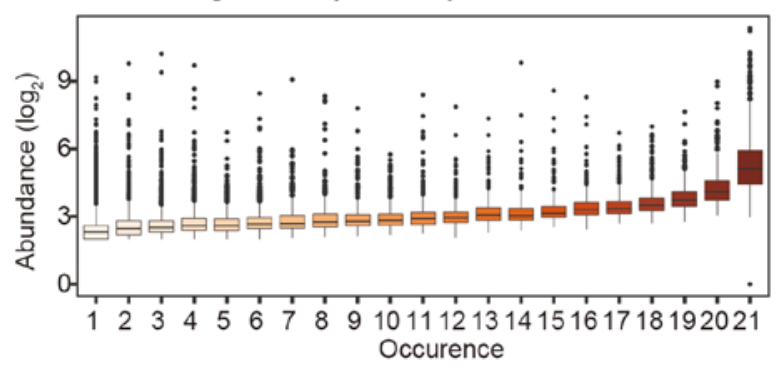

B 12 mouse liver samples

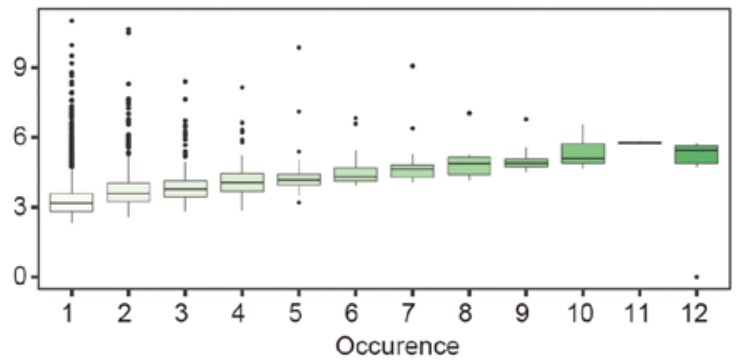

D 12 mouse brain samples

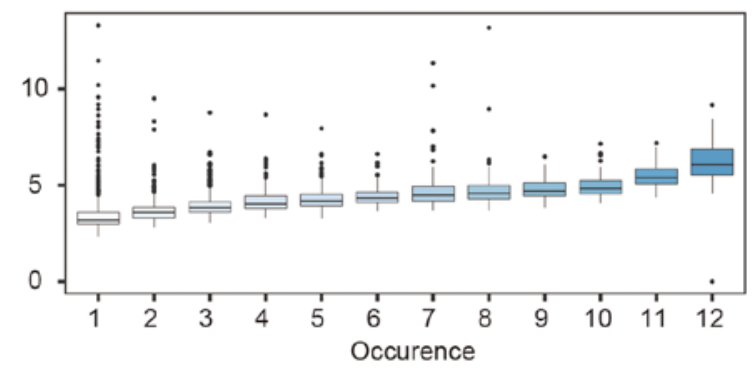

Figure 7. Comparison of the abundance of different circRNA occurrences. $\mathrm{x}$-axis represents the occurrence of circRNAs in the recruited samples; $\mathrm{y}$-axis shows the abundance of circRNAs $\left(\log _{2}\right)$. (A) Thirteen human HCC samples. (B) Twelve mouse liver samples. (C) Seventeen human brain samples. (D) Twelve mouse brain samples. (E) Twenty-one human granulocyte samples. 
A Human $\mathrm{HCC}$ samples

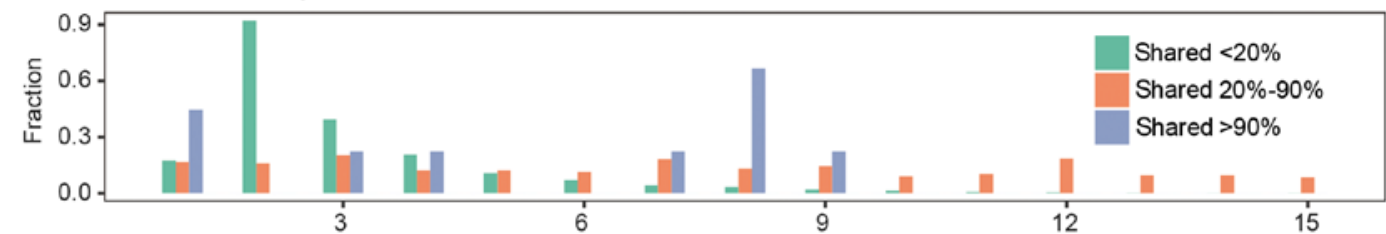

B Mouse liver samples

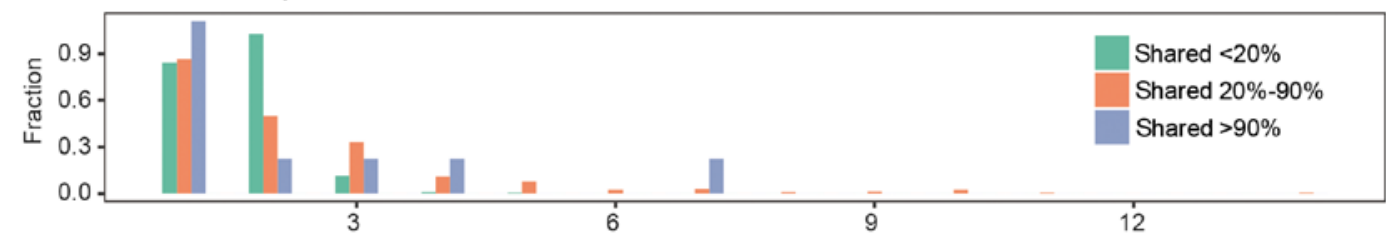

C Human brain samples

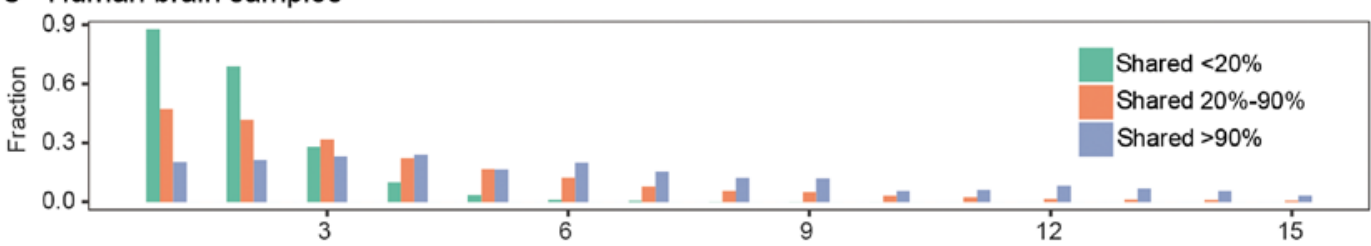

D Mouse brain samples

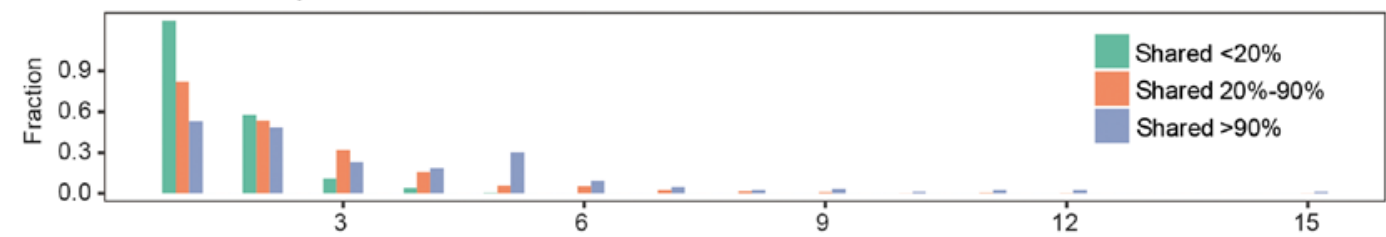

E Human granulocytes samples

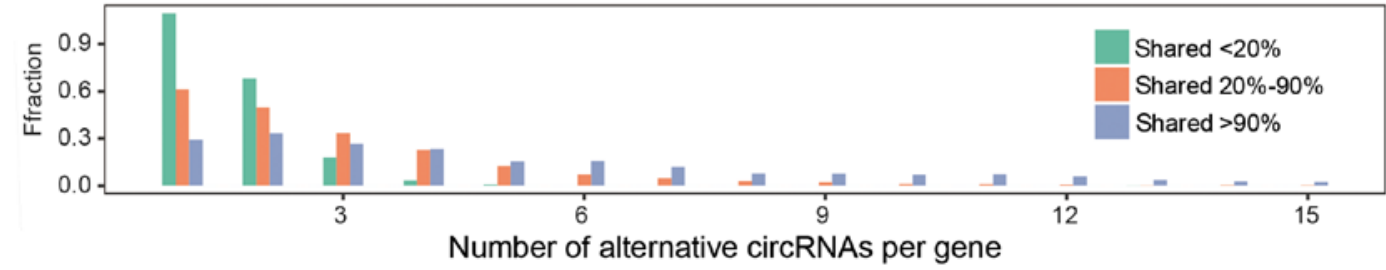

Figure 8. Number of distinct circRNAs per gene locus in humans and mice. $\mathrm{x}$-axis represents the number of alternative circRNAs in a single gene; $\mathrm{y}$-axis shows the fraction of genes. Based on the occurrence of the circRNA gene loci in the recruited samples, these circRNA gene loci were assigned to one of three categories: shared $20 \%$, shared $20-90 \%$, and shared $90 \%$. Shared $20 \%$ are the gene locus-expressed circRNAs in $<20 \%$ of the recruited samples. Shared $20-90 \%$ are the gene locus-expressed circRNAs in $>20 \%$ and $<90 \%$ of the recruited samples. Shared $90 \%$ are gene locus-expressed circRNAs in $>90 \%$ of the recruited samples. (A) Fifty-five human HCC samples. (B) Twelve mouse liver samples. (C) Thirty human brain samples. (D) Twelve mouse brain samples. (E) Twenty-one human granulocyte samples.

occurring circRNA was 5 times more than that of the lowest occurring circRNA. We also analyzed the human granulocyte dataset and found that its circRNA expression profile was similar to that of the human brain samples (Fig. 7).

Then, we discovered that a single gene locus can produce multiple circRNAs. We investigated whether the different occurrences of gene circRNAs corresponded to different numbers of circular isoforms. Based on the occurrence of the circRNA gene loci in the recruited samples, these circRNA gene loci were assigned to one of three categories: shared $20 \%$, shared $20-90 \%$, and shared $90 \%$. Shared $20 \%$ are the gene loci of expressed circRNAs in $<20 \%$ of the recruited samples. Shared $20-90 \%$ are the gene locus-expressed circRNAs in $>20 \%$ and $<90 \%$ of the recruited samples. Shared $90 \%$ are the gene locus-expressed circRNAs in $>90 \%$ of recruited samples. Most of the shared $20 \%$ circRNA gene loci have one or two circRNA isoforms. Shared $90 \%$ circRNA gene loci have multiple circRNA isoforms. A total of 230 of these gene loci gave rise to $>10$ circRNAs in human granulocytes (Fig. 8). These gene loci are circRNA hotspot gene loci (38). The shared $90 \%$ circRNA loci have not only more distinct circRNA isoforms but also highly abundant circRNA isoforms compared to those in the other categories. We then analyzed the alternative splicing and alternative backsplicing of highly shared circRNA gene loci. Typically, the shared circRNA genes express more than two circRNAs in their gene locus; only one or two are highly abundant, whereas the others are lowly abundant and diverse. For example, two of the four circular isoforms of the circRNA UBXN7 were highly abundant. CircRNA UBXN7-1, derived from exon 3-5, was universally expressed in all the human granulocyte samples. CircRNA UBXN7-2 derived from exon 2-5 was detected in 14 of 21 granulocyte samples. Its other two circular isoforms were low abundance and were detected in less than five samples. The alternative backsplicing and alternative cis-splicing of circRNAs were diverse. 


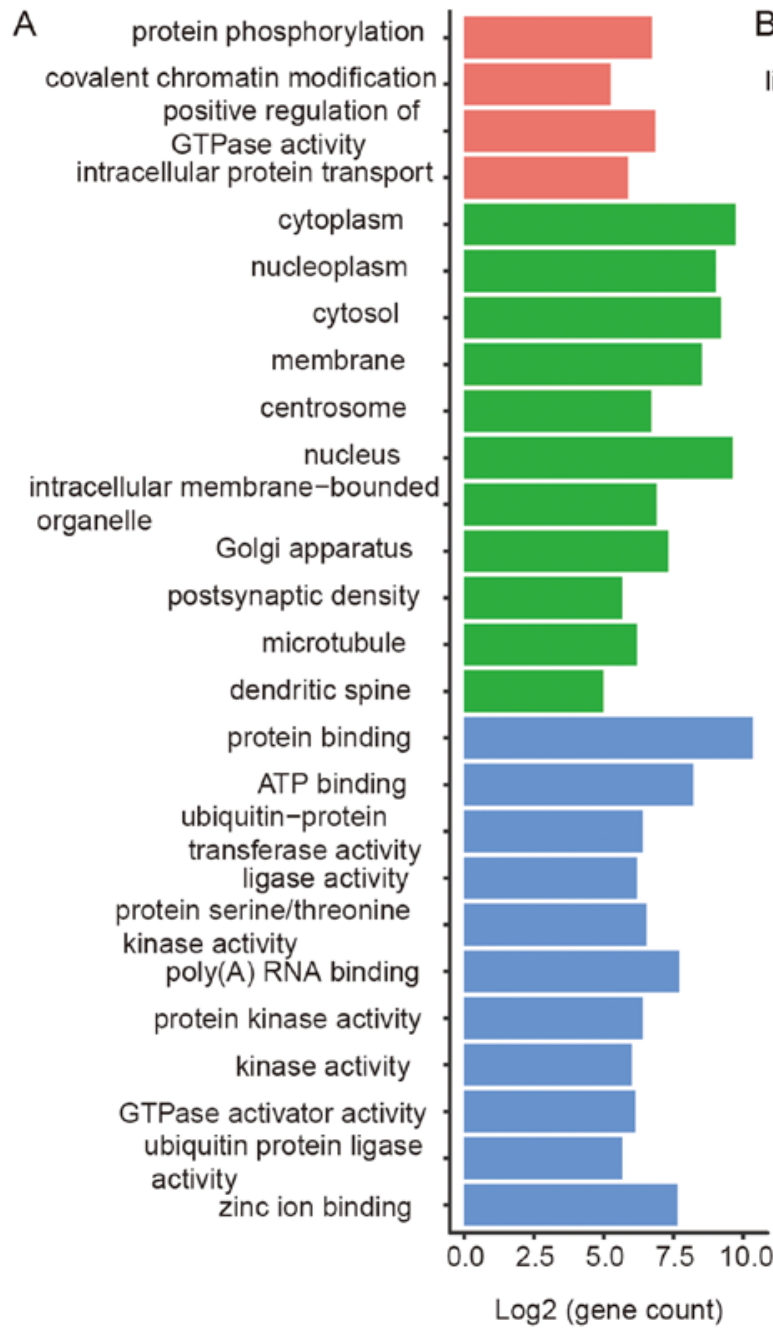

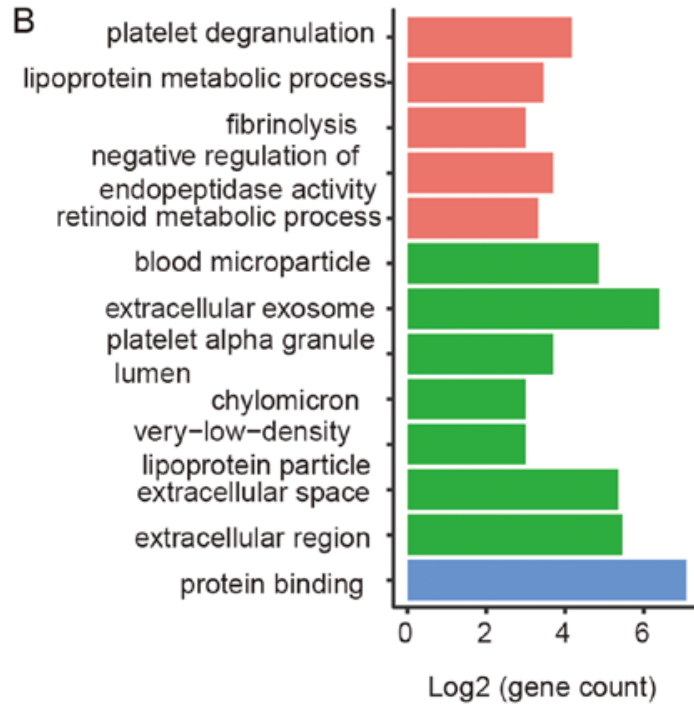

GO term (FDR cuttoff $0.1 \%$ )

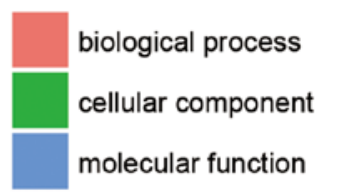

Figure 9. GO analysis of the shared circRNA host genes. (A) Brain and (B) liver. Enriched terms are grouped by GO category: biological process (red), cellular component (green), and molecular function (blue).

Furthermore, we investigated the relative circular to linear transcript abundance. We suggested that the predominant circRNAs have a circular to linear ratio $>1$. We found 2,045 circRNA isoforms that are predominant transcripts among the 55 HCC samples, but only 8 predominant circRNAs, were shared in the HCC samples. Moreover, in the 30 brain samples, there were 5,017 predominant circRNAs and 405 shared circRNAs (Table II). Predominant circRNAs were not significantly enriched in shared circRNAs.

In summary, shared circRNAs are highly abundant. The shared circRNA gene loci have multiple distinct circRNAs. In these shared circRNA gene loci, circRNA expression demonstrates diverse alternative cis-splicing and alternative backsplicing.

Comparison of the tissue-specific shared circRNAs in humans. Even though a large number of circRNAs are inter-individually diverse and vary among the samples, a small proportion of circular RNAs is shared. Previous studies indicated that the expression of circular RNA is related to the genomic origin of the linear transcripts $(5,23,24)$ and that circular RNAs regulate the transcription of host mRNAs $(9,50,51)$. We conducted a Gene Ontology analysis on the linear transcripts derived from the shared liver and brain circRNAs, which revealed significant differences between them. Since there are almost no shared circRNAs in the liver, we considered the circRNAs detected in $>20 \%$ of the samples as shared circRNAs in the liver. We found that liver samples were enriched with lipoprotein metabolic process and extracellular exosome while brain samples were enriched with protein phosphorylation, postsynaptic density, and protein kinase activity (Fig. 9). Both liver and brain samples contained numerous protein binding genes, and most of the GO terms were related to tissue-specific functions. Highly represented gene categories included ApoE and ALB genes in the liver and RIMS1, HTT and KLHL24 in the brain (Fig. 9 and Table III).

Furthermore, we also analyzed the liver, brain, blood, granulocyte and heart shared circRNAs, i.e., 39 shared circRNAs in these tissues (Table III). Some of these shared circRNAs have been previously validated by experiments, e.g., circZKSCAN1 (52) and circMAN1A2 (50,53). CDR1as was detected in the three other tissues except the blood and granulocyte samples.

Conservation of the identified circRNA candidates between human and mouse. Previous research indicated that circular RNAs are evolutionarily conserved in function $(2,23,49,54)$. 
Table III. CircRNAs shared in different tissues.

\begin{tabular}{|c|c|c|c|c|c|c|}
\hline $\operatorname{chr}$ & start & end & gene & transcript & strand & circRNA \\
\hline chr1 & 26729380 & 26774901 & ARID1A & ARID1A-201 & + & chr1:26729650-26732792(+) \\
\hline $\operatorname{chr} 1$ & $1.17 \mathrm{E}+08$ & $1.18 \mathrm{E}+08$ & MAN1A2 & MAN1A2-001 & + & chr1:117402185-117442325(+) \\
\hline $\operatorname{chr} 1$ & $1.17 \mathrm{E}+08$ & $1.18 \mathrm{E}+08$ & MAN1A2 & MAN1A2-001 & + & chr1:117402185-117420649(+) \\
\hline $\operatorname{chr} 1$ & $1.17 \mathrm{E}+08$ & $1.18 \mathrm{E}+08$ & MAN1A2 & MAN1A2-001 & + & chr1:117402185-117414831(+) \\
\hline $\operatorname{chr} 1$ & $1.17 \mathrm{E}+08$ & $1.18 \mathrm{E}+08$ & MAN1A2 & MAN1A2-001 & + & chr1:117402185-117405645(+) \\
\hline $\operatorname{chr} 1$ & 66958911 & 66960078 & MIER1 & MIER1-005 & + & chr1:66958058-66963160(+) \\
\hline $\operatorname{chr} 1$ & 26921726 & 26946862 & NUDC & NUDC-001 & + & chr1:26942659-26943065(+) \\
\hline $\operatorname{chr} 1$ & $1.81 \mathrm{E}+08$ & $1.81 \mathrm{E}+08$ & STX6 & STX6-002 & - & chr1:180984676-180993425(-) \\
\hline $\operatorname{chr} 1$ & 21715124 & 21721146 & USP48 & USP48-008 & - & chr1:21715388-21721764(-) \\
\hline $\operatorname{chr} 10$ & 5709626 & 5714574 & FAM208B & FAM208B-014 & + & chr10:5699524-5714207(+) \\
\hline $\operatorname{chr} 10$ & $1.02 \mathrm{E}+08$ & $1.02 \mathrm{E}+08$ & FBXW4 & FBXW4-002 & - & chr10:101667885-101676436(-) \\
\hline $\operatorname{chr} 12$ & $1.23 \mathrm{E}+08$ & $1.24 \mathrm{E}+08$ & RILPL1 & RILPL1-001 & - & chr12:123498543-123499536(-) \\
\hline $\operatorname{chr} 13$ & 45962176 & 46052759 & ZC3H13 & ZC3H13-002 & - & chr13:46003138-46020557(-) \\
\hline chr14 & 45121588 & 45131261 & FKBP3 & FKBP3-003 & - & chr14:45118027-45130790(-) \\
\hline $\operatorname{chr} 14$ & 49820096 & 49852780 & NEMF & NEMF-005 & - & chr14:49825866-49831361(-) \\
\hline $\operatorname{chr} 14$ & 39179090 & 39182750 & PNN & PNN-005 & + & chr14:39179090-39179462(+) \\
\hline $\operatorname{chr} 14$ & 22909490 & 22911792 & RBM23 & RBM23-012 & - & chr14:22909482-22911403(-) \\
\hline $\operatorname{chr} 14$ & 22905585 & 22919149 & RBM23 & RBM23-004 & - & chr14:22906194-22911403(-) \\
\hline $\operatorname{chr} 18$ & 9136805 & 9235820 & ANKRD12 & ANKRD12-009 & + & chr18:9182381-9221999(+) \\
\hline $\operatorname{chr} 18$ & 21704957 & 21864974 & MIB 1 & MIB1-004 & + & $\operatorname{chr18:21765771-21779685(+)}$ \\
\hline $\operatorname{chr} 19$ & 8461969 & 8465372 & HNRNPM & HNRNPM-014 & + & chr19:8455404-8463686(+) \\
\hline $\operatorname{chr} 2$ & $1.12 \mathrm{E}+08$ & $1.12 \mathrm{E}+08$ & ZC3H6 & ZC3H6-001 & + & chr2:112299848-112300029(+) \\
\hline $\operatorname{chr} 2$ & $1.12 \mathrm{E}+08$ & $1.12 \mathrm{E}+08$ & ZC3H6 & ZC3H6-001 & + & chr2:112299848-112325197(+) \\
\hline $\operatorname{chr} 22$ & 50372072 & 50444391 & PPP6R2 & PPP6R2-004 & + & chr22:50372019-50394135(+) \\
\hline $\operatorname{chr} 3$ & $1.96 \mathrm{E}+08$ & $1.97 \mathrm{E}+08$ & RNF168 & RNF168-001 & - & chr3:196487398-196488683(-) \\
\hline chr3 & $1.58 \mathrm{E}+08$ & $1.59 \mathrm{E}+08$ & RSRC1 & RSRC1-201 & + & $\operatorname{chr} 3: 158122102-158123991(+)$ \\
\hline $\operatorname{chr} 3$ & $1.7 \mathrm{E}+08$ & $1.7 \mathrm{E}+08$ & SEC62 & SEC62-009 & + & chr3:169976945-169988359(+) \\
\hline $\operatorname{chr} 4$ & $1.52 \mathrm{E}+08$ & $1.53 \mathrm{E}+08$ & FBXW7 & FBXW7-004 & - & chr4:152411302-152412529(-) \\
\hline $\operatorname{chr} 4$ & $1.28 \mathrm{E}+08$ & $1.28 \mathrm{E}+08$ & LARP1B & LARP1B-005 & + & chr4:128074459-128077962(+) \\
\hline chr6 & 4836098 & 4954373 & CDYL & CDYL-007 & + & chr6:4891712-4892379(+) \\
\hline chr6 & 18223868 & 18264823 & DEK & DEK-001 & - & chr6:18236451-18258405(-) \\
\hline $\operatorname{chr} 7$ & $1 \mathrm{E}+08$ & $1 \mathrm{E}+08$ & ZKSCAN1 & ZKSCAN1-001 & + & chr7:100023418-100024307(+) \\
\hline chr8 & 61623710 & 61714596 & ASPH & ASPH-001 & - & chr8:61618977-61653660(-) \\
\hline $\operatorname{chr} 8$ & 67083848 & 67195611 & CSPP1 & CSPP1-003 & + & chr8:67131950-67137603(+) \\
\hline $\operatorname{chr} 8$ & $1.08 \mathrm{E}+08$ & $1.08 \mathrm{E}+08$ & EMC2 & EMC2-004 & + & chr8:108449822-108455930(+) \\
\hline chr9 & 5919008 & 6007787 & KIAA2026 & KIAA2026-002 & - & chr9:5968018-5988545(-) \\
\hline chr9 & 33948374 & 33989043 & UBAP2 & UBAP2-004 & - & chr9:33971650-33973237(-) \\
\hline $\operatorname{chr} 9$ & 33994481 & 34048872 & UBAP2 & UBAP2-010 & - & chr9:33986759-34017189(-) \\
\hline $\operatorname{chr} 9$ & 33921693 & 34048901 & UBAP2 & UBAP2-001 & - & chr9:33960825-33989126(-) \\
\hline
\end{tabular}

First, most circRNAs originated from CDS regions, which are evolutionally preserved in the genome. Second, the backsplice sites of circRNAs are conserved. We compared human and mouse conservation of circRNAs with a previously described computational method (55). In total, we identified 169,044 circRNA candidates in 178 human samples and 9,886 circRNA candidates in 24 mouse samples. In human and mouse, there were 3,579 shared conserved gene locus-detected
circRNA candidates. There were 83,389 gene locus-expressed circRNA candidates in humans and 8,615 circRNA candidates in mice.

\section{Discussion}

Eukaryotic circRNAs are a type of less abundant, but biochemically stable, transcripts that are expressed in diverse 
genomic locations. The abundance of circRNAs is $\sim 1-3 \%$ of the level of poly(A) ${ }^{+}$RNAs (49), and most circRNAs exist in low abundance. Therefore, identifying all of the expressed circRNAs is difficult. Each circRNA prediction algorithm brings its own bias and 'blind spots' (22). We solved this problem by combining the distinct algorithms to yield a more trustworthy and sensitive output (34). It was reported that circRNA isoforms from the same host transcript share the same backsplice sites with different internal exons (24). As a result, the internal exon composition of circRNAs cannot simply be predicted using junction exons and linear RNA exon composition $(24,48,56)$. RAISE is designed to detect circRNA internal exon composition and predict circRNA transcript sequences (Fig. 2C). The availability of coverage and splice information helps in the identification of a circRNA and its exon composition. The internal exon usage of circRNAs was used to predict circRNA transcript sequences and investigate circRNA functions.

We used RAISE to compare the circRNA expression profiles in HCC with adjacent non-tumor tissue samples and did not find any significant differences. Even though there is no significant differential expression between tumor and adjacent non-tumor tissue, we observed that circRNAs expression profiles are diverse between individuals and are independent of the linear gene expression. In the case of circRNA expression, this variation means detected or not, whereas for linear gene expression, it represents whether the abundance is high or low. Since the circRNAs are not highly enriched in HCC-affected tissues, we tested whether various other tissues possessed the same expression patterns. In the brain rRNA depleted RNA-seq data, the ratio of shared circRNAs was $0.5 \%$, which is higher than the ratio in the liver but lower than the ratio of the linear gene. The brain and liver samples displayed high inter-individual variation in the expression of circRNAs. We collected 21 granulocytes samples from seven individuals at three time-points to determine whether circRNAs were reproducibly expressed within one individual. The results showed that circRNAs are not reproducible within one individual. Briefly, the circRNAs fall into two categories: the randomly or variably expressed circRNAs, and the robustly expressed circRNAs. The biological roles of either of these types is not yet clear and thus requires functional studies. Several previous studies have highlighted a few circRNAs which are highly abundant and ubiquitously expressed $(1,55)$.

In agreement with previous research, individual circRNA expression seems to be highly stochastic. However, the variable expression of circRNAs in different samples may also trap miRNAs (57). Meanwhile, some online databases, including Arraystar's circRNA target prediction software, Circ2Traits, CircInteractome and CircNet (58-61), have been developed to predict circRNA-miRNA interaction networks. Circ2Traits is a comprehensive database for circular RNAs with potential association with disease and traits (58). CircNet and CircInteractome, both predict the miRNAs target of circRNAs and create the circRNA-miRNA interaction network $(59,60)$.

We also observed that most of the circRNAs are detected in only a few cell types and that they are not as cell-type-specific as mRNAs (55). The variation and diversity of circRNAs expression profiles may be due to the large number of circRNA transcripts expressed at a low level. Interestingly, most of the
circRNAs co-exist with linear RNA transcripts; however, only a small portion of these circRNAs are predominant transcripts (Table II). There are several shared circRNAs among the recruited samples in the same tissue. The shared circRNAs usually have multiple circular isoforms in the gene locus, and these gene loci are circRNA hotspots. We conducted a Gene Ontology analysis on the host genes that gave rise to shared circRNAs and showed that these genes are related to tissuespecific functions.

We used our RAISE pipeline to show that circRNAs have both intra- and inter-individual variations in their expression patterns. Our findings can be helpful in identifying novel circRNAs and designing better therapeutic approaches. Furthermore, according to our suggestions only the robustly expressed circRNAs are a candidate for usage as a biomarker.

\section{Acknowledgements}

The authors acknowledge the authors behind the circRNA detection algorithms for making their source codes publicly available, NCBI SRA and ENCODE online public dataset. This study was supported by Beijing Postdoctoral Research Foundation (no. 2016ZZ-33), and Beijing Municipal Science and Technology Commission Research Fund (no. Z171100000417004).

\section{References}

1. Salzman J, Gawad C, Wang PL, Lacayo N and Brown PO: Circular RNAs are the predominant transcript isoform from hundreds of human genes in diverse cell types. PLoS One 7 (e30733): e30733, 2012.

2. Jeck WR, Sorrentino JA, Wang K, Slevin MK, Burd CE, Liu J, Marzluff WF and Sharpless NE: Circular RNAs are abundant, conserved, and associated with ALU repeats. RNA 19: 141-157, 2013.

3. Nigro JM, Cho KR, Fearon ER, Kern SE, Ruppert JM, Oliner JD, Kinzler KW and Vogelstein B: Scrambled exons. Cell 64: 607-613, 1991.

4. Capel B, Swain A, Nicolis S, Hacker A, Walter M, Koopman P, Goodfellow $\mathrm{P}$ and Lovell-Badge R: Circular transcripts of the testis-determining gene Sry in adult mouse testis. Cell 73: 1019-1030, 1993.

5. Zhang XO, Wang HB, Zhang Y, Lu X, Chen LL and Yang L: Complementary sequence-mediated exon circularization. Cell 159: 134-147, 2014.

6. Ivanov A, Memczak S, Wyler E, Torti F, Porath HT, Orejuela MR, Piechotta M, Levanon EY, Landthaler M, Dieterich C, et al: Analysis of intron sequences reveals hallmarks of circular RNA biogenesis in animals. Cell Rep 10: 170-177, 2015.

7. Kelly S, Greenman C, Cook PR and Papantonis A: Exon skipping is correlated with exon circularization. J Mol Biol 427: 2414-2417, 2015.

8. Barrett SP, Wang PL and Salzman J: Circular RNA biogenesis can proceed through an exon-containing lariat precursor. eLife 4: e07540, 2015.

9. Conn SJ, Pillman KA, Toubia J, Conn VM, Salmanidis M, Phillips CA, Roslan S, Schreiber AW, Gregory PA and Goodall GJ: The RNA binding protein quaking regulates formation of circRNAs. Cell 160: 1125-1134, 2015.

10. Chen LL and Yang L: Regulation of circRNA biogenesis. RNA Biol 12: 381-388, 2015.

11. Salzman J: Circular RNA expression: Its potential regulation and function. Trends Genet 32: 309-316, 2016.

12. Starke S, Jost I, Rossbach O, Schneider T, Schreiner S, Hung LH and Bindereif A: Exon circularization requires canonical splice signals. Cell Rep 10: 103-111, 2015.

13. Szabo L, Morey R, Palpant NJ, Wang PL, Afari N, Jiang C, Parast MM, Murry CE, Laurent LC and Salzman J: Statistically based splicing detection reveals neural enrichment and tissue-specific induction of circular RNA during human fetal development. Genome Biol 16: 126, 2015. 
14. Ahmed I, Karedath T, Andrews SS, Al-Azwani IK, Mohamoud YA Querleu D, Rafii A and Malek JA: Altered expression pattern of circular RNAs in primary and metastatic sites of epithelial ovarian carcinoma. Oncotarget 7: 36366-36381, 2016.

15. Wang K, Singh D, Zeng Z, Coleman SJ, Huang Y, Savich GL, He X, Mieczkowski P, Grimm SA, Perou CM, et al: MapSplice: Accurate mapping of RNA-seq reads for splice junction discovery. Nucleic Acids Res 38: e178, 2010.

16. Memczak S, Jens M, Elefsinioti A, Torti F, Krueger J, Rybak A, Maier L, Mackowiak SD, Gregersen LH, Munschauer M, et al: Circular RNAs are a large class of animal RNAs with regulatory potency. Nature 495: 333-338, 2013.

17. Hoffmann S, Otto C, Kurtz S, Sharma CM, Khaitovich P, Vogel J, Stadler PF and Hackermüller J: Fast mapping of short sequences with mismatches, insertions and deletions using index structures. PLOS Comput Biol 5: e1000502, 2009.

18. Westholm JO, Miura P, Olson S, Shenker S, Joseph B, Sanfilippo P, Celniker SE, Graveley BR and Lai EC: Genome-wide analysis of drosophila circular RNAs reveals their structural and sequence properties and age-dependent neural accumulation. Cell Rep 9: $1966-1980,2014$

19. Gao Y, Wang J and Zhao F: CIRI: An efficient and unbiased algorithm for de novo circular RNA identification. Genome Biol 16: 4, 2015

20. You X and Conrad TO: Acfs: Accurate circRNA identification and quantification from RNA-Seq data. Sci Rep 6: 38820, 2016.

21. Cheng J, Metge F and Dieterich C: Specific identification and quantification of circular RNAs from sequencing data. Bioinformatics 32: 1094-1096, 2016.

22. Szabo L and Salzman J: Detecting circular RNAs: Bioinformatic and experimental challenges. Nat Rev Genet 17: 679-692, 2016.

23. Rybak-Wolf A, Stottmeister C, Glažar P, Jens M, Pino N, Giusti S Hanan M, Behm M, Bartok O, Ashwal-Fluss R, et al: Circular RNAs in the mammalian brain are highly abundant, conserved, and dynamically expressed. Mol Cell 58: 870-885, 2015.

24. You X, Vlatkovic I, Babic A, Will T, Epstein I, Tushev G, Akbalik G, Wang M, Glock C, Quedenau C, et al: Neural circular RNAs are derived from synaptic genes and regulated by development and plasticity. Nat Neurosci 18: 603-610, 2015.

25. Venø MT, Hansen TB, Ven $\varnothing$ ST, Clausen BH, Grebing M Finsen B, Holm IE and Kjems J: Spatio-temporal regulation of circular RNA expression during porcine embryonic brain development. Genome Biol 16: 245, 2015.

26. Tan WL, Lim BT, Anene-Nzelu CG, Ackers-Johnson M, Dashi A See K, Tiang Z, Lee DP, Chua WW, Luu TD, et al: A landscape of circular RNA expression in the human heart. Cardiovasc Res 113: 298-309, 2017.

27. Burd CE, Jeck WR, Liu Y, Sanoff HK, Wang Z and Sharpless NE: Expression of linear and novel circular forms of an INK4/ ARF-associated non-coding RNA correlates with atherosclerosis risk. PLoS Genet 6: e1001233, 2010.

28. Alnasir J and Shanahan HP: Investigation into the annotation of protocol sequencing steps in the sequence read archive. Gigascience 4: 23, 2015.

29. Birney E, Stamatoyannopoulos JA, Dutta A, Guigó R, Gingeras TR, Margulies EH, Weng Z, Snyder M, Dermitzakis ET, Thurman RE, et al; Children's Hospital Oakland Research Institute: Identification and analysis of functional elements in $1 \%$ of the human genome by the ENCODE pilot project. Nature 447: 799-816, 2007

30. Dong H, Zhang L, Qian Z, Zhu X, Zhu G, Chen Y, Xie X, Ye Q, Zang J, Ren Z, et al: Identification of HBV-MLL4 integration and its molecular basis in Chinese hepatocellular carcinoma. PLoS One 10: e0123175, 2015.

31. Kornienko AE, Dotter CP, Guenzl PM, Gisslinger $\mathrm{H}$ Gisslinger B, Cleary C, Kralovics R, Pauler FM and Barlow DP: Long non-coding RNAs display higher natural expression variation than protein-coding genes in healthy humans. Genome Biol 17: 14, 2016

32. Scheckel C, Drapeau E, Frias MA, Park CY, Fak J, ZuckerScharff I, Kou Y, Haroutunian V, Ma'ayan A, Buxbaum JD, et al: Regulatory consequences of neuronal ELAV-like protein binding to coding and non-coding RNAs in human brain. eLife 5: 5,2016.

33. Liu SJ, Nowakowski TJ, Pollen AA, Lui JH, Horlbeck MA, Attenello FJ, He D, Weissman JS, Kriegstein AR, Diaz AA, et al: Single-cell analysis of long non-coding RNAs in the developing human neocortex. Genome Biol 17: 67, 2016.

34. Hansen TB, Venø MT, Damgaard CK and Kjems J: Comparison of circular RNA prediction tools. Nucleic Acids Res 44: e58, 2016.
35. Harrow J, Frankish A, Gonzalez JM, Tapanari E, Diekhans M, Kokocinski F, Aken BL, Barrell D, Zadissa A, Searle S, et al: GENCODE: The reference human genome annotation for The ENCODE Project. Genome Res 22: 1760-1774, 2012.

36. Quinlan AR and Hall IM: BEDTools: A flexible suite of utilities for comparing genomic features. Bioinformatics 26: 841-842, 2010.

37. Zheng Q, Bao C, Guo W, Li S, Chen J, Chen B, Luo Y, Lyu D, Li Y, Shi G, et al: Circular RNA profiling reveals an abundant circHIPK 3 that regulates cell growth by sponging multiple miRNAs. Nat Commun 7: 11215, 2016.

38. Memczak S, Papavasileiou P, Peters O and Rajewsky N: Identification and characterization of circular RNAs as a new class of putative biomarkers in human blood. PLoS One 10: e0141214, 2015.

39. Kroll KW, Mokaram NE, Pelletier AR, Frankhouser DE, Westphal MS, Stump PA, Stump CL, Bundschuh R, Blachly JS and Yan P: Quality control for RNA-Seq (QuaCRS): An integrated quality control pipeline. Cancer Inform 13 (Suppl 3): 7-14, 2014.

40. Chen C, Khaleel SS, Huang $\mathrm{H}$ and Wu CH: Software for preprocessing Illumina next-generation sequencing short read sequences. Source Code Biol Med 9: 8, 2014.

41. Pertea M, Kim D, Pertea GM, Leek JT and Salzberg SL: Transcript-level expression analysis of RNA-seq experiments with HISAT, StringTie and Ballgown. Nat Protoc 11: 1650-1667, 2016.

42. Li H, Handsaker B, Wysoker A, Fennell T, Ruan J, Homer N, Marth G, Abecasis G and Durbin R; 1000 Genome Project Data Processing Subgroup: The Sequence Alignment/Map format and SAMtools. Bioinformatics 25: 2078-2079, 2009.

43. Anders S, Pyl PT and Huber W: HTSeq - a Python framework to work with high-throughput sequencing data. Bioinformatics 31: 166-169, 2015.

44. Pertea M, Pertea GM, Antonescu CM, Chang TC, Mendell JT and Salzberg SL: StringTie enables improved reconstruction of a transcriptome from RNA-seq reads. Nat Biotechnol 33: 290-295, 2015.

45. Huang W, Sherman BT and Lempicki RA: Bioinformatics enrichment tools: Paths toward the comprehensive functional analysis of large gene lists. Nucleic Acids Res 37: 1-13, 2009.

46. Robinson MD, McCarthy DJ and Smyth GK: edgeR: A Bioconductor package for differential expression analysis of digital gene expression data. Bioinformatics 26: 139-140, 2010.

47. Kadota PO, Hajjiri Z, Finn PW and Perkins DL: Precision subtypes of $\mathrm{T}$ cell-mediated rejection identified by molecular profiles. Front Immunol 6: 536, 2015.

48. Zhang XO, Dong R, Zhang Y, Zhang JL, Luo Z, Zhang J, Chen LL and Yang L: Diverse alternative back-splicing and alternative splicing landscape of circular RNAs. Genome Res 26: 1277-1287, 2016

49. Salzman J, Chen RE, Olsen MN, Wang PL and Brown PO: Cell-type specific features of circular RNA expression. PLoS Genet 9: e1003777, 2013.

50. Li Z, Huang C, Bao C, Chen L, Lin M, Wang X, Zhong G, Yu B, $\mathrm{Hu}$ W, Dai L, et al: Exon-intron circular RNAs regulate transcription in the nucleus. Nat Struct Mol Biol 22: 256-264, 2015.

51. Ashwal-Fluss R, Meyer M, Pamudurti NR, Ivanov A, Bartok O, Hanan M, Evantal N, Memczak S, Rajewsky N and Kadener S: circRNA biogenesis competes with pre-mRNA splicing. Mol Cell 56: 55-66, 2014.

52. Liang D and Wilusz JE: Short intronic repeat sequences facilitate circular RNA production. Genes Dev 28: 2233-2247, 2014.

53. Alhasan AA, Izuogu OG, Al-Balool HH, Steyn JS, Evans A, Colzani M, Ghevaert C, Mountford JC, Marenah L, Elliott DJ, et al: Circular RNA enrichment in platelets is a signature of transcriptome degradation. Blood 127: e1-e11, 2016.

54. Thomas LF and Sætrom P: Circular RNAs are depleted of polymorphisms at microRNA binding sites. Bioinformatics 30 : 2243-2246, 2014

55. Guo JU, Agarwal V, Guo H and Bartel DP: Expanded identification and characterization of mammalian circular RNAs. Genome Biol 15: 409, 2014

56. Gao Y, Wang J, Zheng Y, Zhang J, Chen S and Zhao F: Comprehensive identification of internal structure and alternative splicing events in circular RNAs. Nat Commun 7: 12060, 2016.

57. Caiment F, Gaj S, Claessen S and Kleinjans J: High-throughput data integration of RNA-miRNA-circRNA reveals novel insights into mechanisms of benzo[a]pyrene-induced carcinogenicity. Nucleic Acids Res 43: 2525-2534, 2015. 
58. Ghosal S, Das S, Sen R, Basak P and Chakrabarti J: Circ2Traits: A comprehensive database for circular RNA potentially associated with disease and traits. Front Genet 4: 283, 2013.

59. Liu YC, Li JR, Sun CH, Andrews E, Chao RF, Lin FM, Weng SL, Hsu SD, Huang CC, Cheng C, et al: CircNet: A database of circular RNAs derived from transcriptome sequencing data. Nucleic Acids Res 44D: D209-D215, 2016.
60. Dudekula DB, Panda AC, Grammatikakis I, De S, Abdelmohsen K and Gorospe M: CircInteractome: A web tool for exploring circular RNAs and their interacting proteins and microRNAs. RNA Biol 13: 34-42, 2016.

61. Huang M,ZhongZ,Lv M,Shu J,Tian Q and Chen J: Comprehensive analysis of differentially expressed profiles of lncRNAs and circRNAs with associated co-expression and ceRNA networks in bladder carcinoma. Oncotarget 7: 47186-47200, 2016. 